\title{
Impact of tumour RAS/BRAF status in a first-line study of panitumumab + FOLFIRI in patients with metastatic colorectal cancer
}

Meinolf Karthaus*,1, Ralf-Dieter Hofheinz ${ }^{2}$, Laurent Mineur ${ }^{3}$, Henry Letocha ${ }^{4}$, Richard Greil ${ }^{5}$, Josef Thaler ${ }^{6}$, Eva Fernebro ${ }^{7}$, Kelly S Oliner ${ }^{8}$, Michael Boedigheimer ${ }^{9}$, Brian Twomey ${ }^{9}$, Ying Zhang ${ }^{9}$, Gaston Demonty ${ }^{10}$ and Claus-Henning Köhne ${ }^{11}$

\begin{abstract}
${ }^{1}$ Klinikum Neuperlach/Klinikum Harlaching, Oskar-Maria-Graf-Ring 51, D81737 Munich, Germany; ${ }^{2}$ Universitätsmedizin Mannheim, Theodor-Kutzer Ufer 1-3, 68167 Mannheim, Germany; Institut Sainte-Catherine Gl and Liver Cancer Unit, 84000 Avignon, France; ${ }^{4}$ Oncology Clinic, Västmanland's Hospital, 72189 Västerås, Sweden; ${ }^{5}$ Illrd Medical Department, Paracelsus Medical University Salzburg and CCCIT Salzburg Cancer Research Institute, Müllner Hauptstrasse 45, 5020 Salzburg, Austria; ${ }^{6}$ Klinikum WelsGrieskirchen, Grieskirchner Straße 42, A-4600 Wels, Austria; ${ }^{7}$ Central Hospital, Strandvägen 8, 35185 Växjö, Sweden; ${ }^{8}$ Formerly of Amgen Inc., 1 Amgen Center Dr MS 30E-2-C, Thousand Oaks, CA 91320, USA; ${ }^{9}$ Amgen Inc., 1 Amgen Center Dr MS 30E-2-C, Thousand Oaks, CA 91320, USA; ${ }^{10}$ Amgen GmbH, Dammstrasse 23, 6301 Zug, Switzerland and ${ }^{11}$ Onkologie Klinikum Oldenburg, Rahel-Straus-Str. 10, 26133 Oldenburg, Germany
\end{abstract}

Background: To investigate tumour biomarker status and efficacy of first-line panitumumab + FOLFIRI for metastatic colorectal carcinoma $(\mathrm{mCRC})$.

Methods: 154 patients received first-line panitumumab + FOLFIRI every 14 days. Primary end point was objective response rate (ORR). Data were analysed by tumour RAS (KRAS/NRAS) and BRAF status, and baseline amphiregulin (AREG) expression.

Results: Objective responses occurred more frequently in RAS wild type (WT) (59\%) vs RAS mutant (MT) (41\%) mCRC and in RAS WT/BRAFWT (68\%) vs RAS or BRAF MT (37\%) disease. Median response duration was longer in RAS WT (13.0 months) vs RAS MT (5.8 months) (hazard ratio (HR): 0.16). Median progression-free survival was longer in RAS WT vs MT (11.2 vs 7.3 months; HR, 0.37) and was also longer in RAS WT/BRAF WT vs RAS or BRAF MT (13.2 vs 6.9 months; HR, 0.25). Incidence of adverse events was similar regardless of RAS/BRAF status, and no new safety signals were noted. Among patients with RAS WT tumours, ORR was $67 \%$ with high AREG expression and 38\% with low AREG expression.

Conclusions: First-line panitumumab + FOLFIRI was associated with favourable efficacy in patients with RAS WT and RAS WT/ BRAF WT vs MT mCRC tumours and was well tolerated.

FOLFIRI (folinic acid, infusional 5-fluorouracil, and irinotecan) is a recommended first- and second-line chemotherapy backbone in the treatment of metastatic colorectal cancer (mCRC) (National Comprehensive Cancer Network, 2015). Following a recent review by the European Medicines Agency (EMA), both of the epidermal growth factor receptor (EGFR) inhibitors - panitumumab and cetuximab - are now approved in Europe for first-line use in combination with FOLFIRI or FOLFOX (folinic acid, infusional 5-fluorouracil, and oxaliplatin) in patients with RAS wild-type (WT) mCRC.

In the first-line setting, EGFR inhibitors were originally indicated in patients with KRAS WT mCRC in combination with

*Correspondence: Professor M Karthaus; E-mail: meinolf.karthaus@klinikum-muenchen.de

Received 9 May 2016; revised 21 July 2016; accepted 19 September 2016; published online 20 October 2016

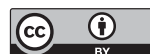

(c) 2016 Cancer Research UK. All rights reserved 0007-0920/16

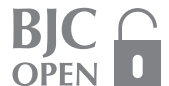


either FOLFOX (panitumumab or cetuximab) or FOLFIRI (cetuximab). Subsequently, mutations were identified in KRAS and NRAS in $17 \%$ of patients with non-mutated KRAS exon 2 in the phase III PRIME trial of panitumumab + FOLFOX $v s$ FOLFOX alone (Douillard et al, 2013). As a result, EGFR inhibitor use was refined to include only those patients with RAS WT disease. At the same time, studies also identified BRAF as an important negative prognostic - though not predictive - marker for survival in patients with mCRC, regardless of treatment (Phipps et al, 2012; Douillard et al, 2013; Peeters et al, 2013, 2014a, c). Tumour expression of the biomarker amphiregulin (AREG) has also been correlated with survival during anti-EGFR therapy (Jacobs et al, 2009; Loupakis et al, 2014).

The improved risk-benefit profile of EGFR inhibitor treatment in patients selected by RAS status was later verified in trials of firstand second-line FOLFOX and FOLFIRI in combination with panitumumab or cetuximab (Douillard et al, 2013; Heinemann et al, 2014a, b; Venook et al, 2014; Bokemeyer et al, 2015; Van Cutsem et al, 2015). Importantly, patients with RAS mutant (MT) tumours showed no improvement in efficacy with the addition of EGFR inhibitor compared with chemotherapy alone. Indeed, there is some evidence that EGFR inhibitors combined with FOLFOX in such patients are detrimental compared with FOLFOX alone (Douillard et al, 2013; Bokemeyer et al, 2015), and EGFR inhibitors should therefore not be given to patients with $R A S$ MT tumours.

Panitumumab plus FOLFIRI demonstrated superiority over FOLFIRI alone in a second-line phase II trial that demonstrated improved progression-free survival (PFS) in patients with RAS WT mCRC, although overall survival (OS) was not significantly different in this study (Peeters et al, 2014b). The only published study of first-line panitumumab plus FOLFIRI in mCRC is a single-arm trial that indicated the efficacy and predictable safety profile of the combination in KRAS WT patients (Köhne et al, 2012), although preliminary data have been presented from a phase II study in which panitumumab plus FOLFOX4 or FOLFIRI was evaluated in patients with KRAS WT colorectal cancer and liverlimited disease (Abad et al, 2014). Here, we present a retrospective analysis of this first-line trial of panitumumab plus FOLFIRI, reporting efficacy and safety data for first-line FOLFIRI plus panitumumab according to tumour RAS/BRAF status and AREG levels in patients with mCRC.

\section{PATIENTS AND METHODS}

Study design. This is a retrospective analysis of data from a phase II, single-arm study (NCT 00508404); full details of the study design have been presented previously (Köhne et al, 2012). In brief, first-line panitumumab $\left(6 \mathrm{mg} \mathrm{kg}^{-1}\right)+$ FOLFIRI were administered every 14 days until progression, unacceptable toxicity or withdrawal of consent. If FOLFIRI was withdrawn or suspended as a result of toxicity, panitumumab could be continued, and vice versa.

Patients. Patients were adults with histologically or cytologically confirmed, radiologically measurable mCRC and an Eastern Cooperative Oncology Group (ECOG) performance status of $0-2$. Patients could be enrolled only if all disease sites were evaluated within 28 days before enrolment, and tissue from the primary or metastatic site was available. Those who had received prior systemic therapy (including anti-EGFR therapy) for mCRC (except adjuvant fluoropyrimidine-based chemotherapy given $\geqslant 6$ months before enrolment) were excluded. Full inclusion and exclusion criteria have been reported previously (Köhne et al, 2012).
The study protocol was approved by the relevant independent ethics committees. The study was conducted in accordance with the regulations and guidelines of the International Conference on Harmonisation of Good Clinical Practice. All patients provided signed, informed consent before any study-related procedures were performed.

End points and analyses. The primary end point was the objective response rate (ORR) assessed using modified Response Evaluation Criteria in Solid Tumors (mRECIST v1.0) (Therasse et al, 2000). Secondary end points included disease control rate (DCR), duration of response (DoR), depth of response (DpR; defined as the percentage of tumour shrinkage at nadir or progression), PFS, and time to progression (TTP). Resection rates were also evaluated, as was early tumour shrinkage (ETS), defined as the percentage of patients with $\geqslant 30 \%$ or $\geqslant 20 \%$ tumour shrinkage at week 8 (exploratory analysis). Safety was evaluated in terms of the incidence and severity of adverse events (AEs), using the National Cancer Institute Common Toxicity Criteria version 3.0.

Data were analysed descriptively by tumour $R A S / B R A F$ status. Tumour specimens were assayed for mutations in KRAS exons 3-4, NRAS exons 2-4 and BRAF exon 15 by bidirectional Sanger sequencing. Mutations in KRAS exon 2 were analysed by CEmarked DxS kit.

Baseline tumour AREG levels were analysed in the RAS WT and MT populations. Total RNA was extracted from formalin-fixed paraffin-embedded tissue samples and AREG expression levels were analysed by qualified reverse transcription quantitative polymerase chain reaction (RT-qPCR) assays (see Supplementary Material for details). A cutoff point for AREG status was prespecified based on analysis of data from an earlier clinical trial (STEPP) (Lacouture et al, 2010). Cox proportional hazards (PH) models were used to evaluate AREG expression levels as a continuous covariate. Decision curves were used to estimate the PFS hazard ratio (HR) with increasing levels of baseline AREG expression. A Gaussian Process (GP) model was used to fit the PH model (Joensuu et al, 2012) using the GPstuff toolkit in MATLAB (Vanhatalo et al, 2013) - details are included in the Supplementary Material.

\section{RESULTS}

Ascertainment of tumour KRAS/BRAF mutation status. In total, 154 patients were enrolled in the study (Köhne et al, 2012). One patient withdrew consent and three patients had no DNA and/or tumour tissue available. The RAS mutational analysis therefore included 150 patients, of whom 143 received at least one dose of panitumumab.

Overall, $38 \%$ of patients had KRAS exon 2 mutations, and $10 \%$ had RAS mutations beyond KRAS exon 2 (KRAS exon 3 and 4 mutations each in $3 \%$ of tumours; NRAS exon 2 and 3 mutations each in $2 \%$ of tumours; no tumour was found to carry an NRAS exon 4 mutation). Complete RAS data were available for 143 of 150 patients, of whom 69 (45\%) had RAS WT tumours (i.e., WT for exons 2, 3 and 4 of both KRAS and NRAS). BRAF mutations were present in nine patients (6\%), all of whom had tumours that were WT for RAS.

Patients. Baseline characteristics were generally well balanced between patients with RAS WT vs RAS MT mCRC, and between patients with RAS WT/BRAF WT and RAS or BRAF MT status (Table 1). More patients with RAS WT and RAS WT/BRAF WT mCRC had liver-limited metastases, whereas more RAS MT and $R A S$ or BRAF MT patients had metastases at other sites only. The sum of the longest diameters of measurable target lesions was also slightly larger in patients with RAS WT and RAS WT/BRAF WT mCRC compared with the corresponding MT populations. The median follow-up time in the study was 34.0 weeks (range, 5-223 weeks). 
Efficacy. Overall, 141 patients were evaluable for response assessment. Objective responses occurred more frequently in patients with RAS WT (59\%) vs RAS MT (41\%) mCRC, and in patients with RAS WT/BRAF WT (68\%) vs RAS or BRAF MT (37\%) tumours (Table 2). Disease control rates were similar between patients with RAS WT (91\%) and MT (92\%) mCRC and were slightly higher in the RAS WT/BRAF WT (95\%) group than in those with RAS or BRAF MT (89\%) mCRC (Table 2).

The DoR analysis included only those patients who experienced a partial or complete response: 40 patients with mCRC WT for both $R A S$ and $B R A F$, and 30 patients with MT RAS and WT BRAF mCRC (no patient with BRAF MT disease responded to treatment). Median DoR was longer in RAS WT (13.0 months) vs RAS MT (5.8 months) disease (HR, 0.16 (95\% confidence interval (CI): 0.07, 0.37)) (Figure 1).

Median DpR $(n=141)$ was also significantly greater in patients with RAS WT (59.3\% (Q1, Q3: 26.4, 77.0\%)) than in those with
RAS MT $(35.7 \%(18.8,62.0 \%) ; P=0.0181)$ disease. Early tumour shrinkage $\geqslant 30 \%$ was reported in $49 \%$ of patients with RAS WT disease and $37 \%$ of those with RAS MT disease (odds ratio, 1.6), while ETS $\geqslant 20 \%$ was reported in 74 and $50 \%$ of patients, respectively (odds ratio: 2.8). Early tumour shrinkage was associated with longer PFS in the RAS WT vs MT groups ( $\geqslant 30 \%$ cutoff: median 14.3 vs 7.8 months, HR, 0.29 ; $\geqslant 20 \%$ cutoff: 13.3 vs 7.3 months, HR, 0.34). Furthermore, when patient groups were combined $(n=135)$, patients achieving ETS had significantly longer PFS than those not achieving the relevant ETS criteria (ETS $\geqslant 30 \%$ vs $<30 \%$ : median 10.9 vs 7.2 months, $\mathrm{HR}, 0.45, P=0.0003$; ETS $\geqslant 20 \%$ vs <20\%: 9.1 vs 6.9 months, HR, $0.48, P=0.0005)$.

Metastasis resection rates $(n=143)$ were numerically higher in patients with RAS WT ( $n=9$; $13 \%$ (95\% CI: 6.1, 23.3)) vs RAS MT mCRC $(n=7 ; 9 \%(3.9,18.5))$ and were also higher in patients with RAS WT/BRAF WT $(n=9 ; 15 \%(7.1,26.6))$ vs those with RAS or $B R A F$ MT status $(n=7 ; 8 \%(3.5,16.6))$. Rates of complete

Table 1. Baseline demographics and disease characteristics

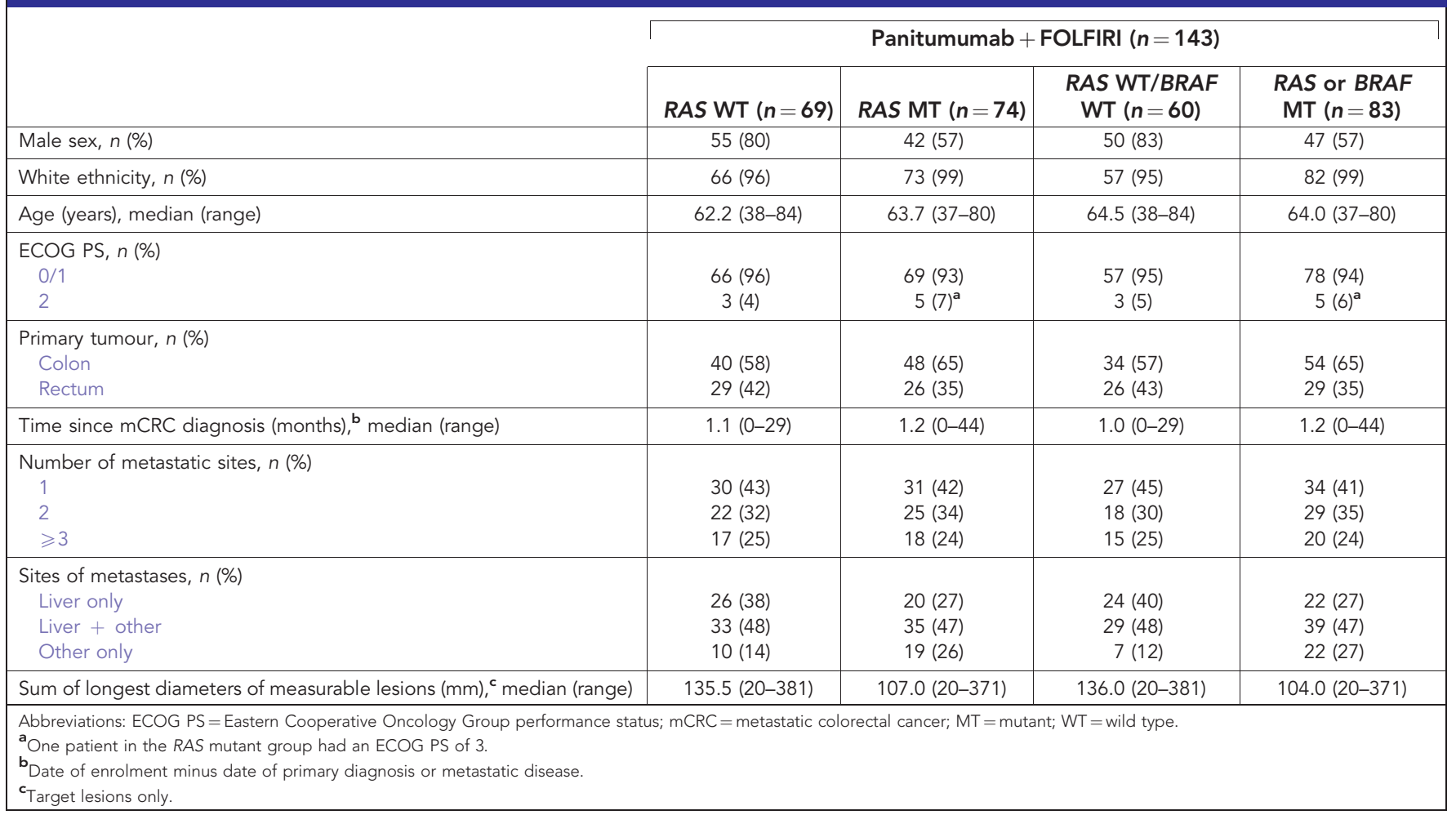

Table 2. Best response, objective response and disease control rates

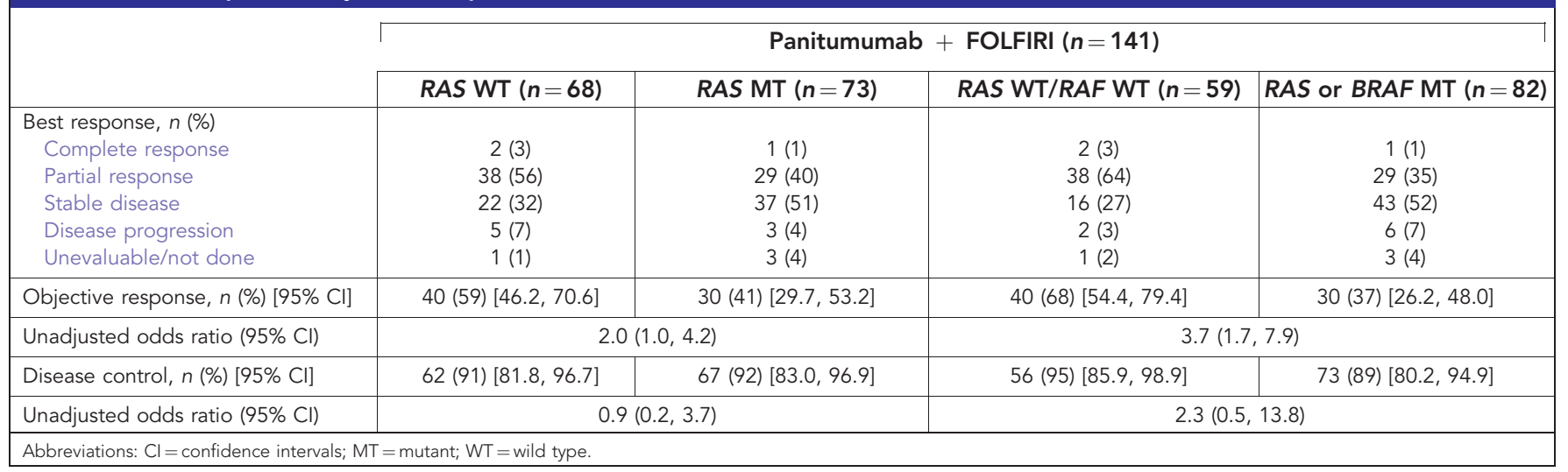




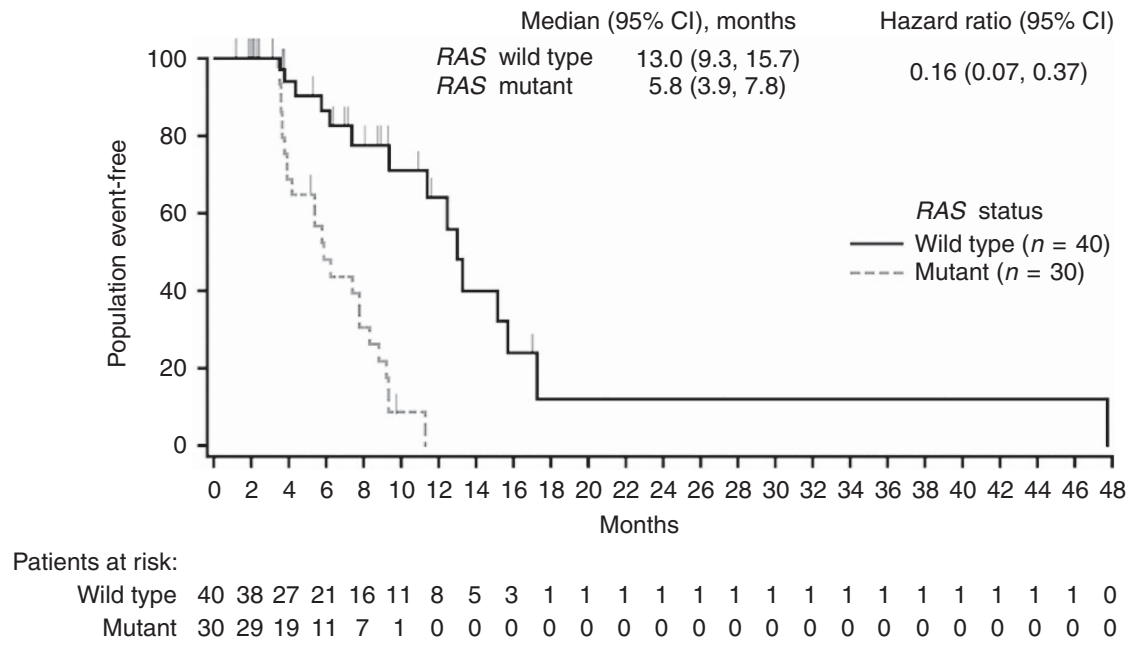

Figure 1. Duration of response by tumour RAS status.

resection were similar across the four groups (RAS WT, $n=4,6 \%$ (95\% CI: 1.6, 14.2); RAS MT, $n=5,7 \%(2.2,15.1) ; R A S \mathrm{WT} / B R A F$ WT, $n=4,7 \%(1.9,16.2)$; RAS or BRAF MT, $n=5,6 \%(2.0,13.5))$.

Median PFS $(n=143)$ was longer in patients with RAS WT vs MT status (11.2 vs 7.3 months, respectively; HR, 0.37 (95\% CI: $0.24,0.58)$ ) (Figure $2 \mathrm{~A}$ ) and was also longer in patients with RAS WT/BRAF WT vs RAS or BRAF MT mCRC (13.2 vs 6.9 months, respectively; HR, 0.25 (95\% CI: 0.15, 0.41)) (Figure 2B). Median TTP $(n=143)$ was longer in patients with RAS WT $(13.2$ (95\% CI: 7.8, 17.0) months) vs MT tumours (7.3 (95\% CI: 6.1, 7.6) months) and in those with RAS WT/BRAF WT (13.3 (95\% CI: 9.0, 17.0) months) vs RAS or BRAF MT tumours (7.2 (95\% CI: 5.7, 7.4) months).

Median overall survival $(n=143)$ was not reached in any of the four $R A S / B R A F$ groups, although the number of deaths was numerically lower in the RAS WT $(n=5 ; 7 \%)$ and $R A S \mathrm{WT} / B R A F$ WT $(n=4 ; 7 \%)$ groups $v$ s the RAS MT $(n=11 ; 15 \%)$ and RAS or BRAF MT $(n=12 ; 14 \%)$ groups (Cox proportional HR, RAS WT vs $R A S \mathrm{MT}=0.42$ (95\% CI: 0.15, 1.23); RAS WT/BRAF WT vs RAS or $B R A F \mathrm{MT}=0.37$ (95\% CI: $0.12,1.15)$ ).

Safety. For patients with RAS WT tumours $(n=69)$, the median (range) cumulative dose of panitumumab delivered (adjusted for weight) was $72.0(6.0-395.5) \mathrm{mg} \mathrm{kg}^{-1}$. The median (range) relative dose intensity (RDI) was 89\% (35-101). For patients with RAS MT tumours $(n=74)$, the median (range) adjusted cumulative panitumumab dose was $60.4(5.8-150.0) \mathrm{mg} \mathrm{kg}^{-1}$, with a median (range) RDI of 90\% (45-103). In the RAS WT/BRAF WT and MT groups, the median (range) adjusted cumulative panitumumab dose was $74.8(6.0,395.5)$ and $58.0(5.8,150.0) \mathrm{mg} \mathrm{kg}^{-1}$, respectively, while the median (range) RDI was $89 \%$ (35-101) and $90 \%$ (45-103), respectively.

The incidence of AEs was similar regardless of tumour RAS/ $B R A F$ status (Table 3 ), and no new safety signals were noted in these analyses. AEs leading to study discontinuation occurred in $24-32 \%$ of patients across RAS/BRAF groups (Table 3), and treatment-related AEs leading to study discontinuation occurred in $16-27 \%$ of patients across $R A S / B R A F$ groups (Table 3 ).

Skin toxicity of any grade occurred in 68 of 69 patients (99\%) with RAS WT tumours, 71 of 74 patients (96\%) with RAS MT tumours, 59 of 60 patients (98\%) with RAS WT/BRAF WT tumours and 80 of 83 patients (96\%) with RAS or BRAF MT tumours.

Amphiregulin expression. Of the 154 patients in the study, 100 had evaluable RAS status and AREG levels (RAS WT, $n=50 ; R A S$ MT, $n=50$ ) (Supplementary Table S1). Tumours with high AREG levels more commonly had WT RAS (31 of 50;62\%) while those with low AREG levels more commonly had MT RAS (36 of 50; $72 \%)$ (Supplementary Table S2). Among RAS WT patients, the ORR $(95 \%$ CI) was $67 \%(51,82)$ in those with high AREG expression and $38 \%(18,58)$ in those with low AREG expression (difference: 29\% $(2,53)$ ) (Supplementary Table S3). ORRs in the RAS MT group were $31 \%(10,53)$ and $45 \%(29,60)$, respectively (difference, $-14 \%(-39,15))$. The HR for PFS was more favourable in the high AREG group (RAS WT/RAS MT: 0.30 (95\% CI: 0.12, 0.75)) compared with the low AREG group (0.49 (95\% CI: 0.21 , 1.1)) (Supplementary Figure S1).

The Cox PH model showed a significant RAS-by-AREG interaction $(P=0.03)$ (Supplementary Table S4). There was a steep transition between responders and non-responders as AREG expression decreased, which was mainly the result of changes in the RAS WT group (Supplementary Figure S2).

\section{DISCUSSION}

This analysis represents the first reported RAS data beyond KRAS exon 2 for panitumumab + FOLFIRI in the first-line treatment of $\mathrm{mCRC}$, an indication that has recently been approved by the EMA. The results show consistently favourable efficacy for first-line panitumumab + FOLFIRI treatment in patients with RAS WT/ $B R A F$ WT tumours compared with MT mCRC tumours. This is consistent with the primary data for the KRAS analysis of this study (Köhne et al, 2012), in which response rates for patients with KRAS WT $(n=86)$ and KRAS MT $(n=68)$ tumours were 56 and $38 \%$ and median PFS was 8.9 and 7.2 months, respectively. In the current analysis, extended RAS testing identified 69 patients with RAS WT tumours, and small increases in response rate (59\%) and median PFS (11.2 months) were seen in these patients compared with the primary analysis population. The results are also consistent with previous RAS analysis data for panitumumab plus FOLFIRI in the treatment of mCRC (Cohn et al, 2011; Mitchell et al, 2011; Peeters et al, 2014b; Abad et al, 2014). Thus, patients whose tumours harbour RAS mutations beyond KRAS exon 2 are unlikely to benefit from addition of panitumumab to FOLFIRI. Consistent results have also been reported in studies with another anti-EFGR monoclonal antibody, cetuximab (Van Cutsem et al, 2015; Heinemann et al, 2014a, b), highlighting the importance of up-front tumour RAS testing in patients being considered for EGFR inhibitor therapy. As OS was neither an end point nor followed in this trial, efficacy of panitumumab + FOLFIRI was consistent with that reported for RAS WT populations in first-line 
A

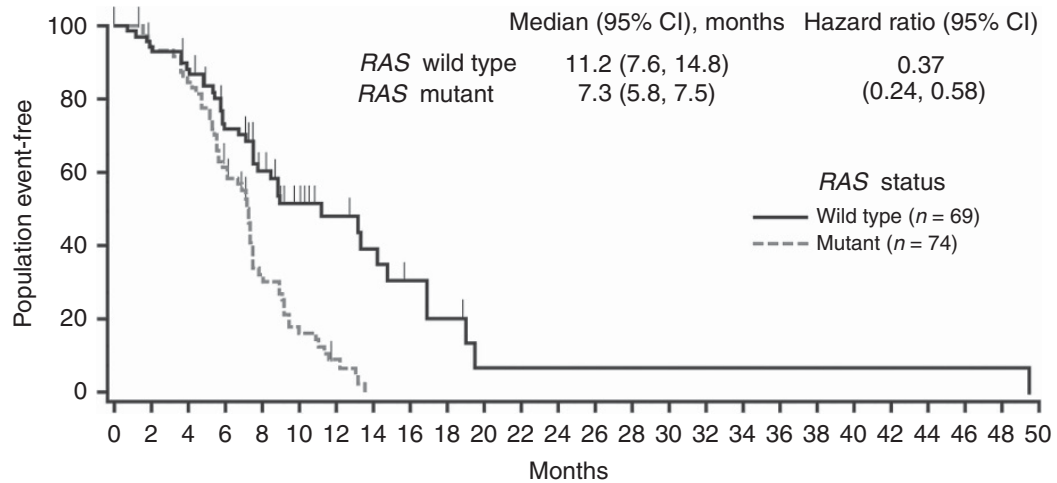

Patients at risk:

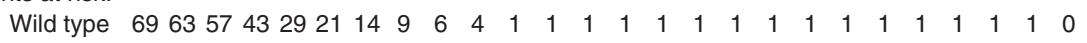

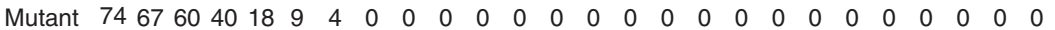

B

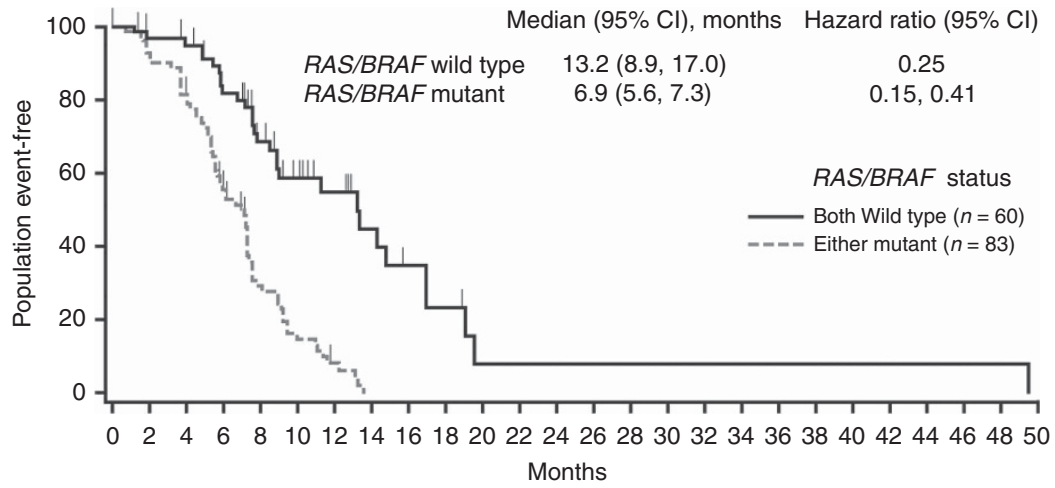

Patients at risk:

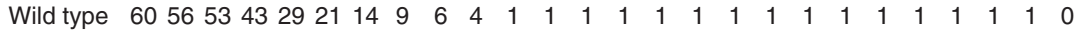

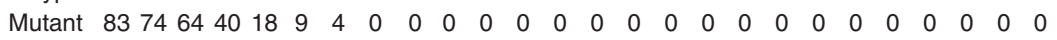

Figure 2. Progression-free survival by tumour (A) RAS and (B) RAS/BRAF status.

\begin{tabular}{|c|c|c|c|c|}
\hline & \multicolumn{4}{|c|}{ Panitumumab + FOLFIRI $(n=143)$} \\
\hline & $\begin{array}{l}\text { RAS WT } \\
(n=69)\end{array}$ & $\begin{array}{l}\text { RAS MT } \\
(n=74)\end{array}$ & $\begin{array}{c}\text { RAS WT/RAF } \\
\text { WT }(n=60)\end{array}$ & $\begin{array}{c}\text { RAS or BRAF } \\
\text { MT }(n=83)\end{array}$ \\
\hline $\begin{array}{l}\text { Any } \mathbf{A E}, \boldsymbol{n}(\%) \\
\text { Worst grade } \geqslant 3 \\
\text { Serious } A E \\
\text { AEs leading to discontinuation }{ }^{\text {a }}\end{array}$ & $\begin{array}{l}69(100) \\
59(86) \\
40(58) \\
21(30)\end{array}$ & $\begin{array}{l}74(100) \\
57(77) \\
38(51) \\
18(24)\end{array}$ & $\begin{array}{l}60(100) \\
52(87) \\
32(53) \\
19(32)\end{array}$ & $\begin{array}{l}83(100) \\
64(77) \\
46(55) \\
20(24)\end{array}$ \\
\hline $\begin{array}{l}\text { Any treatment-related } \mathbf{A E}, \boldsymbol{n}(\%) \\
\text { Worst grade } \geqslant 3 \\
\text { Serious } \mathrm{AE} \\
\text { AEs leading to discontinuation }{ }^{\mathrm{a}}\end{array}$ & $\begin{array}{l}69(100) \\
50(72) \\
20(29) \\
16(23)\end{array}$ & $\begin{array}{l}74(100) \\
50(68) \\
18(24) \\
13(18)\end{array}$ & $\begin{array}{l}60(100) \\
44(73) \\
14(23) \\
16(27)\end{array}$ & $\begin{array}{l}83(100) \\
56(67) \\
24(29) \\
13(16)\end{array}$ \\
\hline
\end{tabular}

studies of panitumumab + FOLFOX4 (Douillard et al, 2013; Schwartzberg et al, 2014; Douillard et al, 2015), as well as in studies of cetuximab in combination with FOLFIRI or FOLFOX4 (Van Cutsem et al, 2015; Bokemeyer et al, 2011; Ciardiello et al, 2014). For example, in the CRYSTAL study of FOLFIRI plus cetuximab, the PFS in patients with WT RAS (11.4 months) and MT RAS (7.4 months) (Van Cutsem et al, 2015) was almost identical to that in the present study (11.2 and 7.3 months, respectively). The results of the present study are also consistent with previous data showing that BRAF mutations are associated with poor prognosis in mCRC regardless of first-line treatment (Phipps et al, 2012; Morris et al, 2014). Discussion is ongoing, however, regarding the potential usefulness of EGFR inhibitors in patients with RAS WT/BRAF MT mCRC, Two recent meta-analyses, have reached differing conclusions regarding the predictive role of $B R A F$ mutations in patients receiving EGFR inhibitor therapy. While Pietrantonio et al (2015) focus on the lack of significant ORR, PFS or OS benefits on addition of EGFR inhibitors to chemotherapy, Rowland et al (2015), who included trials of first- and secondline treatment, conclude that there is insufficient evidence to demonstrate a different treatment benefit between patients with RAS WT/BRAF WT and RAS WT/BRAF MT disease, and therefore insufficient data to exclude patients with $R A S$ WT/BRAF MT from EGFR inhibitor therapy. Regardless, such 
patients should therefore be considered to be at high risk of rapid progression and should be managed accordingly.

No new safety signals were seen with the combination of panitumumab + FOLFIRI in RAS WT/BRAF WT population; AEs were similar to those seen in the KRAS exon 2 WT population of this study (Thaler et al, 2012) and in previous studies using this combination in patients with mCRC (Cohn et al, 2011; Mitchell et al, 2011; Peeters et al, 2014b). Overall 28\% of patients withdrew from study treatment because of AEs. Consistent with existing data on EGFR inhibitors, there was a high incidence of skin toxicity. While there was no protocol-mandated proactive management of skin toxicity in the present study, it is now recommended for patients receiving EGFR inhibitors (Boone et al, 2007; Melosky et al, 2009). An earlier analysis of data from the present study showed a higher incidence of skin toxicity in patients with KRAS WT tumours than in those with KRAS MT tumours (Thaler et al, 2012), which may reflect the higher mean cumulative panitumumab dose and longer duration of treatment (i.e., panitumumab cycles) received by the KRAS WT group. However, exposure-adjusted AE rates showed integument-related toxicity overall to be higher in the KRAS MT vs WT population. Thus there is no evidence of an association between tumour $K R A S / R A S$ status and toxicity. Furthermore, despite the high incidence of skin toxicity, generic quality of life (QoL) instruments have shown no impact of EGFR inhibitors plus FOLFIRI on overall QoL (Melosky et al, 2009; Bennett et al, 2011; Thaler et al, 2012). Although proactive management of skin toxicity may have reduced the impact for patients, it may also be that the QoL tools used in the study provided too general an assessment to determine the true impact of this AE. Future trials of EGFR inhibitors should include skin-toxicity specific QoL assessment tools to support better understanding of the true impact of this AE on patient wellbeing.

Among patients with RAS WT mCRC, high AREG expression was associated with response to panitumumab + FOLFIRI. Consistent with other studies (Jacobs et al, 2009; Baker et al, 2011; Pentheroudakis et al, 2013; Loupakis et al 2014; Jonker et al, 2014; Stahler et al,2016), there was an interaction between RAS and AREG levels. A higher percentage of patients with RAS WT mCRC had high AREG levels compared with those with RAS MT mCRC, suggesting that AREG levels are associated with EGFR signalling. Further studies are needed to determine whether there is an AREG expression level below which there is little or no response to panitumumab + FOLFIRI treatment. Biomarker studies remain critical to our understanding of targeted agents in mCRC, and warrant further investigation.

One of the strengths of this comprehensive study was the high percentage of patients from the original cohort who were available for $R A S / B R A F$ testing - which was conducted centrally for all specimens - allowing the identification of additional risks for lack of response to treatment. It should be noted that the analyses were retrospective and exploratory in nature, although, as noted above, the results were consistent with previous analyses of efficacy by RAS mutation status in patients with mCRC.

In conclusion, first-line panitumumab + FOLFIRI was associated with consistently favourable efficacy in patients with RAS WT/BRAF WT vs MT mCRC tumours and was well tolerated, despite the expected high incidence of skin toxicity. The combination of first-line panitumumab + FOLFIRI also gave efficacy similar to that seen in the RAS WT populations in other first-line studies of EGFR-targeted agents plus FOLFIRI or FOLFOX. As per the licensed indication for panitumumab, therefore, patients with RAS mutations should not receive panitumumab treatment. Across all lines of therapy, determination of tumour RAS status improves identification of patients unlikely to respond to treatment with panitumumab compared with evaluation of KRAS exon 2 alone. The combination of panitumumab with
FOLFIRI can be considered as an important treatment option for first-line patients with RAS WT/BRAF WT mCRC.

\section{ACKNOWLEDGEMENTS}

This work was supported by Amgen (Europe) GmbH. Medical writing support (funded by Amgen (Europe) $\mathrm{GmbH}$ ) was provided by Dan Booth PhD (Bioscript Medical Ltd).

\section{CONFLICT OF INTEREST}

MK is an advisor for, and has received honoraria from, Amgen Ltd. $\mathrm{R}-\mathrm{DH}$ has received honoraria from Amgen Ltd. LM is an advisor for, and has received honoraria and research funding from, Amgen. HL has acted as a consultant/advisor for Amgen Ltd \& Roche Ltd. RG has received research support from Amgen. JT has received honoraria and research funding from Amgen Ltd. KSO is a former employee of Amgen Inc. MB, BT, YZ, GD are employees of Amgen Inc. C-HK has acted as a consultant/advisor to Amgen Ltd, Merck KG Darmstadt \& Roche Ltd. EF declares no conflict of interest.

\section{REFERENCES}

Abad A, Massuti B, Gravalos C, Escudero P, Guillen C, Manzano JL, Gomez MA, Safont MJ, Plazas JG, Sastre J, Pericay C, Dueñas R, López-López C, Losa F, Ayerbes MV, González E, Yuste A, Carrato A, Aranda E (2014) Phase II trial of panitumumab plus FOLFOX4 or FOLFIRI in subjects with KRAS wild-type colorectal cancer and liver-limited disease: the PLANET study. J Clin Oncol 32(5 Suppl): Abstract 3560.

Baker JB, Dutta D, Watson D, Maddala T, Munneke BM, Shak S, Rowinsky EK, Xu LA, Harbison CT, Clark EA, Mauro DJ, Khambata-Ford S (2011) Tumour gene expression predicts response to cetuximab in patients with KRAS wildtype metastatic colorectal cancer. Br J Cancer 104: 488-495.

Bennett L, Zhao Z, Barber B, Zhou X, Peeters M, Zhang J, Xu F, Wiezorek J, Douillard JY (2011) Health-related quality of life in patients with metastatic colorectal cancer treated with panitumumab in first- or secondline treatment. Br J Cancer 105: 1495-1502.

Bokemeyer C, Bondarenko I, Hartmann JT, de Braud F, Schuch G, Zubel A, Celik I, Schlichting M, Koralewski P (2011) Efficacy according to biomarker status of cetuximab plus FOLFOX-4 as first-line treatment for metastatic colorectal cancer: the OPUS study. Ann Oncol 22: 1535-1546.

Bokemeyer C, Köhne CH, Ciardiello F, Lenz HJ, Heinemann V, Klinkhardt U, Beier F, Duecker K, van Krieken JH, Tejpar S (2015) FOLFOX4 plus cetuximab treatment and RAS mutations in colorectal cancer. Eur J Cancer 51: 1243-1252.

Boone SL, Rademaker A, Liu D, Pfeiffer C, Mauro DJ, Lacouture ME (2007) Impact and management of skin toxicity associated with antiepidermal growth factor receptor therapy: survey results. Oncology 72: $152-159$.

Ciardiello F, Lenz H-J, Kohne CH, Heinemann V, Tejpar S, Esser R, Beier F, Stroh C, Duecker K, Van Cutsem E (2014) Effect of KRAS and NRAS mutational status on first-line treatment with FOLFIRI plus cetuximab in patients with metastatic colorectal cancer (mCRC): new results from the CRYSTAL trial. J Clin Oncol 32(Suppl 3): Abstract \#LBA443.

Cohn AL, Shumaker GC, Khandelwal P, Smith DA, Neubauer MA, Mehta N, Richards D, Watkins DL, Zhang K, Yassine MR (2011) An open-label, single-arm, phase 2 trial of panitumumab plus FOLFIRI as second-line therapy in patients with metastatic colorectal cancer. Clin Colorectal Cancer 10: 171-177.

Douillard JY, Douillard JY, Oliner KS, Siena S, Tabernero J, Burkes R, Barugel M, Humblet Y, Bodoky G, Cunningham D, Jassem J, Rivera F, Kocákova I, Ruff P, Błasińska-Morawiec M, Śmakal M, Canon JL, Rother M, Williams R, Rong A, Wiezorek J, Sidhu R, Patterson SD (2013) Panitumumab-FOLFOX4 treatment and RAS mutations in colorectal cancer. N Engl J Med 369: 1023-1034. 
Douillard JY, Siena S, Peeters M, Koukakis R, Terwey JH, Tabernero J (2015) Impact of early tumour shrinkage and resection on outcomes in patients with wild-type RAS metastatic colorectal cancer. Eur J Cancer 51: 1231-1242.

Heinemann V, Modest D, Fischer von Weikersthal L, Decker T, Kiani A, Vehling-Kaiser U, Al-Batran S-E, Heintges T, Lerchenmüller C, Kahl C, Seipelt G, Kullmann F, Stauch M, Scheithauer W, Held S, Giessen C, Möhler M, Jagenburg A, Stintzing S (2014a) Independent radiological evaluation of objective response by early tumor shrinkage, and depth of response in FIRE-3 (AIO KRK-0306). Ann Oncol 25(Suppl 2): iil17 Abstract O-0030.

Heinemann V, von Weikersthal LF, Decker T, Kiani A, Vehling-Kaiser U, Al-Batran SE, Heintges T, Lerchenmüller C, Kahl C, Seipelt G, Kullmann F, Stauch M, Scheithauer W, Hielscher J, Scholz M, Müller S, Link H, Niederle N, Rost A, Höffkes HG, Moehler M, Lindig RU, Modest DP, Rossius L, Kirchner T, Jung A, Stintzing S (2014b) FOLFIRI plus cetuximab versus FOLFIRI plus bevacizumab as first-line treatment for patients with metastatic colorectal cancer (FIRE-3): a randomised, openlabel, phase 3 trial. Lancet Oncol 15: 1065-1075.

Jacobs B, De Roock W, Piessevaux H, Van Oirbeek R, Biesmans B, De Schutter J, Fieuws S, Vandesompele J, Peeters M, Van Laethem JL, Humblet Y, Pénault-Llorca F, De Hertogh G, Laurent-Puig P, Van Cutsem E, Tejpar S (2009) Amphiregulin and epiregulin mRNA expression in primary tumors predicts outcome in metastatic colorectal cancer treated with cetuximab. J Clin Oncol 27: 5068-5074.

Joensuu H, Vehtari A, Riihimaki J, Nishida T, Steigen SE, Brabec P, Plank L, Nilsson B, Cirilli C, Braconi C, Bordoni A, Magnusson MK, Linke Z, Sufliarsky J, Federico M, Jonasson JG, Dei Tos AP, Rutkowski P (2012) Risk of recurrence of gastrointestinal stromal tumour after surgery: an analysis of pooled population-based cohorts. Lancet Oncol 13: 265-274.

Jonker DJ, Karapetis CS, Harbison C, O'Callaghan CJ, Tu D, Simes RJ, Malone DP, Langer C, Tebbutt N, Price TJ, Shapiro J, Siu LL, Wong RP, Bjarnason G, Moore MJ, Zalcberg JR, Khambata-Ford S (2014) Epiregulin gene expression as a biomarker of benefit from cetuximab in the treatment of advanced colorectal cancer. Br J Cancer 110: 648-655.

Köhne CH, Hofheinz R, Mineur L, Letocha H, Greil R, Thaler J, Fernebro E, Gamelin E, Decosta L, Karthaus M (2012) First-line panitumumab plus irinotecan/5-fluorouracil/leucovorin treatment in patients with metastatic colorectal cancer. J Cancer Res Clin Oncol 138: 65-72.

Lacouture ME, Mitchell EP, Piperdi B, Pillai MV, Shearer H, Iannotti N, Xu F, Yassine M (2010) Skin toxicity evaluation protocol with panitumumab (STEPP), a phase II, open-label, randomized trial evaluating the impact of a pre-Emptive Skin treatment regimen on skin toxicities and quality of life in patients with metastatic colorectal cancer. J Clin Oncol 28: 1351-1357.

Loupakis F, Cremolini C, Fioravanti A, Orlandi P, Salvatore L, Masi G, Schirripa M, Di Desidero T, Antoniotti C, Canu B, Faviana P, Sensi E, Lupi C, Fontanini G, Basolo F, Di Paolo A, Danesi R, Falcone A, Bocci G (2014) EGFR ligands as pharmacodynamic biomarkers in metastatic colorectal cancer patients treated with cetuximab and irinotecan. Target Oncol 9: 205-214

Melosky B, Burkes R, Rayson D, Alcindor T, Shear N, Lacouture M (2009) Management of skin rash during EGFR-targeted monoclonal antibody treatment for gastrointestinal malignancies: Canadian recommendations. Curr Oncol 16: 16-26.

Mitchell EP, Piperdi B, Lacouture ME, Shearer H, Iannotti N, Pillai MV, Xu F, Yassine M (2011) The efficacy and safety of panitumumab administered concomitantly with FOLFIRI or Irinotecan in second-line therapy for metastatic colorectal cancer: the secondary analysis from STEPP (Skin Toxicity Evaluation Protocol With Panitumumab) by KRAS status. Clin Colorectal Cancer 10: 333-339.

Morris V, Overman MJ, Jiang ZQ, Garrett C, Agarwal S, Eng C, Kee B, Fogelman D, Dasari A, Wolff R, Maru D, Kopetz S (2014) Progression-free survival remains poor over sequential lines of systemic therapy in patients with BRAF-mutated colorectal cancer. Clin Colorectal Cancer 13: 164-171.

National Comprehensive Cancer Network (2015) NCCN Clinical Practice Guidelines in Oncology. Colon Cancer. V2.2015. Jamestown, PA: National Comprehensive Cancer Network.

Peeters M, Oliner KS, Parker A, Siena S, Van Cutsem E, Huang J, Humblet Y, Van Laethem JL, André T, Wiezorek J, Reese D, Patterson SD (2013) Massively parallel tumor multigene sequencing to evaluate response to panitumumab in a randomized phase III study of metastatic colorectal cancer. Clin Cancer Res 19: 1902-1912.

Peeters M, Oliner KS, Price TJ, Cervantes A, Sobrero AF, Ducreux M, Hotko Y, Andre T, Chan E, Lordick F, Punt CJA, Strickland A, Wilson G, Ciuleanu TE, Roman L, Van Cutsem E, Yu H, Jung AS, Sidhu R, Patterson SD (2014a) Updated analysis of KRAS/NRAS and BRAF mutations in study 20050181 of panitumumab (pmab) plus FOLFIRI for second-line treatment (tx) of metastatic colorectal cancer (mCRC). J Clin Oncol 32(Suppl 5): Abstract \#3568.

Peeters M, Oliner KS, Price TJ, Cervantes A, Sobrero AF, Ducreux M, Hotko Y, Andre T, Chan E, Lordick F, Punt CJA, Strickland A, Wilson G, Ciuleanu T-E, Roman L, Van Cutsem E, Tian Y, Jung AS, Sidhu R, Patterson SD (2014c) Analysis of KRAS/NRAS mutations in phase 3 study 20050181 of panitumumab (pmab) plus FOLFIRI versus FOLFIRI for second-line treatment (tx) of metastatic colorectal cancer (mCRC). J Clin Oncol 32(Suppl 3): Abstract \#LBA387.

Peeters M, Price TJ, Cervantes A, Sobrero A, Ducreux MP, André T, Lordick F, Punt CJA, Koukakis R, Terwey J, van Custem E (2014b) Tumour shrinkage and response outcomes during second-line panitumumab (pmab) + FOLFIRI vs FOLFIRI treatment. Ann Oncol 25(Suppl 4): Abstract \#7739 (546P).

Pentheroudakis G, Kotoula V, De Roock W, Kouvatseas G, Papakostas P, Makatsoris T, Papamichael D, Xanthakis I, Sgouros J, Televantou D, Kafiri G, Tsamandas AC, Razis E, Galani E, Bafaloukos D, Efstratiou I, Bompolaki I, Pectasides D, Pavlidis N, Tejpar S, Fountzilas G (2013) Biomarkers of benefit from cetuximab-based therapy in metastatic colorectal cancer: interaction of EGFR ligand expression with RAS/RAF, PIK3CA genotypes. BMC Cancer 13: 49.

Phipps AI, Buchanan DD, Makar KW, Burnett-Hartman AN, Coghill AE, Passarelli MN, Baron JA, Ahnen DJ, Win AK, Potter JD, Newcomb PA (2012) BRAF mutation status and survival after colorectal cancer diagnosis according to patient and tumor characteristics. Cancer Epidemiol Biomarkers Prev 21: 1792-1798.

Pietrantonio F, Cremolini C, Petrelli F, Di Bartolomeo M, Loupakis F, Maggi C, Antoniotti C, de Braud F, Falcone A, Iacovelli R (2015) First-line anti-EGFR monoclonal antibodies in panRAS wild-type metastatic colorectal cancer: A systematic review and meta-analysis. Crit Rev Oncol Hematol 96: 156-166.

Rowland A, Dias MM, Wiese MD, Kichenadasse G, McKinnon RA, Karapetis CS, Sorich MJ. Meta-analysis of BRAF mutation as a predictive biomarker of benefit from anti-EGFR monoclonal antibody therapy for $R A S$ wild-type metastatic colorectal cancer (2015) Br J Cancer 112: 1888-1894.

Schwartzberg LS, Rivera F, Karthaus M, Fasola G, Canon JL, Hecht JR, Yu H, Oliner KS, Go WY (2014) PEAK: a randomized, multicenter phase II study of panitumumab plus modified fluorouracil, leucovorin, and oxaliplatin (mFOLFOX6) or bevacizumab plus mFOLFOX6 in patients with previously untreated, unresectable, wild-type KRAS exon 2 metastatic colorectal cancer. J Clin Oncol 32: 2240-2247.

Stahler A, Heinemann V, Giessen-Jung C, Crispin A, Schalhorn A, Stintzing S, Fischer von Weikersthal L, Vehling-Kaiser U, Stauch M, Quietzsch D, Held S, von Einem JC, Holch J, Neumann J, Kirchner T, Jung A, Modest DP (2016) Influence of mRNA expression of epiregulin and amphiregulin on outcome of patients with metastatic colorectal cancer treated with 5FU/LV plus irinotecan or irinotecan plus oxaliplatin as first-line treatment (FIRE 1-trial). Int J Cancer 138: 739-746.

Thaler J, Karthaus M, Mineur L, Greil R, Letocha H, Hofheinz R, Fernebro E, Gamelin E, Baños A, Köhne CH (2012) Skin toxicity and quality of life in patients with metastatic colorectal cancer during first-line panitumumab plus FOLFIRI treatment in a single-arm phase II study. BMC Cancer 12: 438.

Therasse P, Arbuck SG, Eisenhauer EA, Wanders J, Kaplan RS, Rubinstein L, Verweij J, Van Glabbeke M, van Oosterom AT, Christian MC, Gwyther SG (2000) New guidelines to evaluate the response to treatment in solid tumors. European Organization for Research and Treatment of Cancer, National Cancer Institute of the United States, National Cancer Institute of Canada. J Natl Cancer Inst 92: 205-216.

Van Cutsem E, Lenz HJ, Kohne CH, Heinemann V, Tejpar S, Melezínek I, Beier F, Stroh C, Rougier P, van Krieken JH, Ciardiello F (2015) Fluorouracil, leucovorin, and irinotecan plus cetuximab treatment and RAS mutations in colorectal cancer. J Clin Oncol 33: 692-700.

Vanhatalo J, Riihimäki J, Hartikainen J, Jylänki P, Tolvanen V, Vehtari A (2013) GPstuff: Bayesian modeling with Gaussian processes. J Mach Learn Res 14: 1179. 
Venook AP, Niedzwiecki D, Lenz H-J, Innocenti F, Mahoney MR, O’Neil BH, Shaw JE, Polite BN, Hochster HS, Atkins JN, Goldberg RM, Mayer RJ, Schilsky RL, Bertagnolli MM, Blanke CD. Cancer and Leukemia Group B (Alliance), SWOG, and ECOG (2014) CALGB/SWOG 80405: Phase III trial of irinotecan/5-FU/leucovorin (FOLFIRI) or oxaliplatin/5-FU/ leucovorin (mFOLFOX6) with bevacizumab (BV) or cetuximab (CET) for patients (pts) with KRAS wild-type (wt) untreated metastatic adenocarcinoma of the colon or rectum (MCRC). J Clin Oncol 32(5 Suppl): Abstract LBA3.

(c) (i) This work is licensed under the Creative Commons Attribution 4.0 International License. To view a copy of this license, visit http://creativecommons.org/licenses/by/4.0/

Supplementary Information accompanies this paper on British Journal of Cancer website (http://www.nature.com/bjc) 